\title{
RUSSELL: DESCRIPCION Y EXISTENCIA
}

WONFILIO TREJO

Universidad Nacional

Autónoma de México.

El presente trabajo intenta contribuir al esclarecimiento de las relaciones entre el pensamiento de Meinong y el de Russell, en el marco de la teoría de las descripciones definidas, y destacar la idea ontológica de existencia que se sustenta en dicha teoría. Este propósito es el que guía la exposición que se hace de la teoría.

En esta primera parte mantendremos que la doctrina de Meinong sobre los objetos no existentes no viola en sí misma el principio de no contradicción, que el problema que esta doctrina ofrece a la teoría de las descripciones definidas no es tanto un problema lógico, aunque así haya parecido a Russell, cuanto un problema ontológico, y estableceremos lo que, a nuestro parecer, son las dos tesis capitales en que descansa la teoría de las descripciones: la tesis epistemológica y la tesis lógica.

Como se sabe, la primera exposición de la teoría de las descripciones de Russell apareció, con gran resonancia, en su artículo On Denoting, publicado en la revista Mind en 1905. La teoría estaba elaborada para responder a ciertas e inevitables dificultades que se derivaban de la doctrina de Meinong dentro de cuyos principios se sostiene, entre otras cosas, que frases tales como "el cuadrado redondo". "la montaña de oro", en contra de cualquier prejuicio empirísta, se refieren a objetos que no son precisamente objetos existentes. En la doctrina de Meinong se introduce una dis- 
tinción precisa entre el objeto en cuanto objeto y el objeto existente. El objeto en cuanto tal sólo consistiría en sus determinaciones esenciales (si cabe decirlo así, pues uno de los principios de Meinong es el de la "independencia del ser determinado, sosein, respecto del ser" que se refiere no sólo a los objetos que no existen, sino también a los que no pueden existir $\mathbf{u}$ objetos imposibles), de manera que la existencia no interviene en lo más mínimo en la definición de lo que es el objeto. Tales determinaciones deben definir el objeto en cuanto objeto si éste ha de ser "algo" y no un puro no-ser, pues de lo contrario no constituirían objeto alguno, si bien tiene que admitirse, en el sentido de Meinong, que las determinaciones esenciales expresadas por las frases antes aludidas no remiten a objetos existentes. Estas frases, por lo tanto, no sỏlo tendrían que remitir a objetos no-existentes, sino que, una vez establecido que las determinaciones de la redondez y la cuadratura pertenecen al cuadrado redondo, lo áureo y lo montañoso a la montaña de oro, podemos formular proposiciones verdaderas acerca de tales objetos. Podemos decir, en efecto, "la montaña de oro es áurea y montañosa", "el cuadrado redondo es redondo y cuadrado".

La situación que aquí se plantea es de las que se prestan a penosas confusiones, porque, según Meinong, de los objetos nombrados por aquellas frases o desarrollados por esas proposiciones, no puede decirse que existan, pero tampoco puede decirse que se reduzcan simplemente a no-ser. Son objetos con propiedades de naturaleza bien definida, de los cuales, sin embargo, no podemos afirmar que existen. El propio Meinong expresó ya esta dificultad al decir, aparentemente en una forma contradictoria, que "hay objetos respecto de los cuales es válido que no hay tales objetos"."

La principal objeción que Russell tiene que hacer a Meinong se origina en el contexto de las declaraciones del tipo

1 Para lo que se asienta en líneas anteriores, y esta cita, cf. J. N. Findlay, Meinong's Theory of Objects and Values, Oxford, 1963, pp. 43-44, 48-49. 
que acabamos de citar. En efecto, sostener que hay objetos que no existen y, no obstante, suponer que son objetos, es tanto como afirmar que hay objetos para los cuales el principio de no contradicción no rige, o negar que ellos estén sujetos a dicho principio. ${ }^{2}$ Según Russell esta afirmación es $\tan$ absolutamente inaceptable como lo sería la afirmación "el cuadrado redondo es redondo y, asimismo, no redondo", en el supuesto de que se admitiera que hay una entidad o un objeto de tales propiedades que, además, no existe. Russell mantendrá, entonces, no que los objetos en cuestión se hallen más allá del principio de no contradicción, sino que pertenecen a su dominio, es decir, que se prestan a infringir el principio, de tal manera que cuando deseamos comprenderlos encontramos que no podemos com. prenderlos como objetos. La situación que plantea Meinong es, según esto, decididamente "intolerable"; es la situación que la teoría de las descripciones tiende no a resolver, sino a eliminar. ${ }^{3}$

Sin embargo, para la "teoría de los objetos" de Meinong la supuesta contradicción de los "objetos no existentes" no parecía representar un problema, o no venía al caso, si se parte de que el pensamiento acerca de un objeto que no existe y el pensamiento acerca de un objeto existente son, para Meinong, totalmente diferentes, de tal suerte que la noción de "ser" resulta también en cada caso distinta. Por lo tanto, cuando digo que "algo" no existe, lo que niego o excluyo de la existencia debe ser algo a lo que impongo esta negación o excluyo de la existencia actual. Algo a lo que impongo la exclusión de la existencia es, así, algo que no tiene lugar en el mundo actual de las existencias. Por ejemplo, los fantasmas no existen, pero si niego que existan no niego, para contradecirme, algo que es parte del mundo actual de las existencias, sino el objeto en cuanto objeto

2 Cf. B. Russell "On Denoting", en Readings in Philosophical Analysis, eds. Feigl and Sellars, New York, 1949, p. 107. (On Denoting se citará con las iniciales O. D.).

3 O. D., p. 106. 
fantasmal posible de tales o cuales propiedades. ${ }^{4}$ Desde este punto de vista el dicho de Meinong de que "hay objetos respecto de los cuales es válido que no hay tales objetos" deja de ser contradictorio, si el "hay" ha de significar, en primer lugar, mera subsistencia, pero no existencia actual, y si el "no hay" debe significar privación de existencia actual, en segundo lugar, pero no privación de subsistencia.

Estas serían las razones por las que Meinong se mantuvo en el convencimiento de que, aun cuando no exista la montaña de oro, la expresión "la montaña de oro" sigue haciendo referencia a un objeto, es decir, no puede carecer de significación y es capaz de intervenir como sujeto de una proposición verdadera. Ahora bien, ya que "hay" objetos de esta naturaleza de los cuales puede afirmarse que "no los hay" en el sentido de referirse a un objeto realmente existente, la proposición "la montaña de oro no existe" no sólo resulta ser significativa, sino que no entraña contradicción en la doctrina de Meinong.

Se puede pensar entonces que la dificultad que le ofrece a Russell la doctrina de los "objetos no existentes" de Meinong no consiste tanto en que estos objetos amenazan el principio de no contradicción (que no lo hacen, dentro de la doctrina de Meinong), cuanto en la circunstancia de que tales objetos puedan subsistir. Hay un pasaje de Ru-

\footnotetext{
4 Véase J. N. Findlay, Op. cit., pp. 54-55. Nicholas Rescher (cf. de este autor "On the logic of existence and denotation" en Philosophical Review, Vol. 69, $\mathrm{N}^{\circ} 2$, Abril 1959, pp. 172 y 176) afirma que "la condición esencial" bajo la cual un objeto $n$ es un objeto no existente posible se representa, mediante el uso adecuado de las modalidades, en la siguiente oración:$$
\sim(\exists x)(x=n) \cdot \mathrm{P}(\exists x)(x=n)
$$

en la que se expresa que, en el sentido del cuantificador existencial, "no existe nada idéntico a $n$, aunque es posible que exista". Pero el mismo autor advierte que el significado del cuantificador existencial es equívoco. En la misma dirección de la distinción de Meinong de que venimos tratando, observa que en la fórmula " $(\exists \mathrm{y}) \quad(\mathrm{y}=\mathrm{x}) \rightarrow \mathbf{E x}$ " (donde $E$ significa existencia real) no sabriamos si el cuantificador existencial está usado para hablar de objetos que existen realmente o de objetos que solamente subsisten como entia imaginationis. En el primer caso la fórmula sería verdadera, y entonces, para evitar el equívoco, propone $(\exists, y) \quad(y=x) \rightarrow E x$; en el segundo caso sería falsa, lo que quedaría representado por $(\exists, y)(y=x) \rightarrow E x$.
} 
ssell en el que se ocupa del principal argumento de Meinong que induce a creerlo así: "Árgüia: si decís que la montaña de oro no existe, es obvio que hay algo que estáis diciendo que no existe, es decir, la montaña de oro; por lo tanto, la montaña de oro debe subsistir en algún oscuro mundo pla. tónico del ser, porque, de otro modo, vuestra afirmación de que la montaña de oro no existe no tendría significado. Confieso que, hasta que di con la teoría de las descripciones, me pareció convincente este argumento". El pasaje confirma la distinción que hemos establecido entre el "hay" de lo que subsiste y el "no hay" de lo que no existe, de cuya unión, en la afirmación "hay la montaña de oro no existente", no puede resultar una contradicción. Pero ahora el argumento de Meinong resulta inconvincente, no porque conduzca a contradicciones, sino porque conduce al platonismo, a la postulación de entes subsistentes en un inundo distinto al de las existencias actuales.

Aclaremos, antes de pasar adelante, este nuestro primer resultado. La doctrina de los "objetos no existentes" no es contradictoria dentro de la teoría misma de Meinong. Si Russell llegó a juzgarla así inicialmente en el artículo $O n$ Denoting se debe, pensamos, o a que no es preciso al exponer a Meinong - pues ahí donde debería haber dicho de los objetos subsistentes que no existen afirma que, para Meinong, "tales objetos no subsisten"_, " o a que al hacer la crítica de aquella teoría ya opera desde fuera una concepción distinta de lo que debe entenderse por "objeto". En todo caso, como se desprende de nuestra última cita, tan pronto como Russell se sitúa dentro del pensamiento de Meinong resulta que el verdadero problema es ontológico: lo constituyen las subsistencias mismas que no existen en tanto que objetos supuestamente denotados por frases como "la montaña de oro"."

5 B. Russell, My Philosophical Development, London, Allen \& Unwin, 1959, p. 84. (Se citará con las iniciales M. PH. D.)

6 O. D., pp. 106-107.

7 Thomas Moro Simpson, quien también considera que la teoría de los objetos no existentes (subsistentes) viola el principio de no contradicción, 
La teoría de las descripciones de Russell va a señalar a partir de aquí que la doctrina de Meinong acerca de los objetos no existentes descansa en una doble confusión cuya aclaración es necesaria para que pueda hacerse manifiesto también cómo no resultan posibles tales objetos. La primera consiste en confundir frases que sólo pueden significar en tanto que forman parte de una proposición con simbolos que significan por sí mismos independientemente de cualquier contexto proposicional, lo que, como veremos, no es más que una forma de confundir "nombre propio" y "descripción". La segunda consiste en confundir el sujeto gramátical de una oración en que aparece, como tal sujeto, una frase descriptiva con el sujeto lógico de una proposición propiamente dicha, o sea, en confundir la función lógica

plantea la difícil cuestión en los términos siguientes en su libro Formas lógicas, realidad y significado (Buenos Aires, Editorial Eudeba, 1964, Cap. III, pp. 98-99) : “"el cuadrado redondo que no es redondo' designa un objeto irreal; si simbolizamos esta expresión con la letra ' $x$ ' tendríamos en. tonces que los enunciados ' $x$ es redondo' $y$ ' $x$ no es redondo' son ambos analíticos, y por lo tanto verdaderos, lo cual viola claramente el principio de contradicción... ambos enunciados serían verdaderos en virtud del principio de identidad que asegura la verdad de todo enunciado de la forma ' $\mathrm{XY}$ es $\mathrm{X}$ ', pero violarían el principio de no contradicción". Pero advirtamos que si ambos enunciados son analíticamente verdaderos, dado lo que ' $x$ ' designa, entonces sólo quiere decir que hay, en el sentido de que subsiste, el cuadrado redondo que no es redondo. $O$ sea, si ambos enunciados son verdaderos, entendido que su verdad no se refiere a ninguna existencia real, sino a una subsistencia, entonces la subsistencia en cuestión es justo la del objeto imposible: el cuadrado redondo que no es redondo. En este sentido la verdad analítica de ambos enunciados se limita a afirmar la subsistencia de una entidad contradictoria: lıay (subsiste) el cuadrado redondo que no es redondo, pero éste no es actual ni posible, sino imposible. Esto es Meinong. Por lo tanto, más que una violación ¿no se trataría tan sólo de afirmar la analiticidad del mismo principio de contradicción? (Sobre la analiticidad de una contradicción, Cf. de Mario Bunge, The Myth of Simplicity, PrenticeHall, Englewood Cliffs, N. J., 1963, 1, 2, p. 24.).

La violación del principio de no contradicción por anbos enunciados sólo podría plantearse en el caso de que se pensase que hay, en el sentido de que existe, algo que corresponde a lo afirmado por ambos enunciados, y que el principio de no contradicción excluye como posible. Pero en este sentido no podría plantearse la violación, porque entonces habría que apelar a los valores de verdad como casos de sustitución de los enunciados; y es claro que los referidos enunciados son de los que no tienen casos de sustitución ni verdaderos ni falsos, como más adelante lo ve el propio Simpson, "pues para ello se requeriria la existencia de un objeto que satisficiera una condición imposible de cumplir" (p. 235). Los subrayados son míos. 
de la frase descriptiva con la función lógica del nombre propio. Ambas confusiones y correspondientes aclaraciones se hallan estrechamente vinculadas. Pero el examen que realiza Russell de la primera confusión trata de señalar que cuando una frase descriptiva, que sólo significa en el contexto de la oración en que aparece, se toma por un sím. bolo que significa por sí mismo, el resultado es o un enunciado falso o un enunciado carente de sentido, mientras que su distinción permite formular enunciados que pueden ser falsos o verdaderos, pero nunca necesariamente falsos ni carentes de sentido. La aclaración de esta primera confusión y la distinción que de ahí resulta constituyen la prueba de que unos símbolos, los nombres propios, tienen una función epistemológica totalmente diferente de la que desempeñan las frases descriptivas; se prueba, en efecto, cómo su distinción hace posible saber a qué se refieren y cómo se refieren, y qué podemos esperar desde el punto de vista del conocimiento de unos y otros símbolos. En cambio, el examen de la segunda confusión muestra cómo de ella resultan enunciados lógicamente inadecuados, engañosos, mientras que su distinción permite formular enunciados de una estructura lógica correcta. En efecto, el análisis mostrará que la frase descriptiva se reduce en último término a un argumento indeterminado de una función proposicional que podría luego satisfacer un valor determinado, esto es, un nombre propio, mientras que su confusión toma el argumento formal por un valor determinado. A la luz de estos señalamientos será posible determinar después el sentido preciso que tiene en Russell el término "existencia".

Establezcamos, entonces, para volver en detalle sobre ellas, las dos tesis esenciales aquí apuntadas hacia las cuales converge una y otra vez la teoría de las descripciones definidas. Primera, que frases descriptivas tales como "la montaña de oro", si bien pueden contribuir al significado de las oraciones en las cuales se presentan, consideradas aisladamente no tienen ningún significado. Segunda, que esas mismas frases pueden aparecer gramáticalmente como sujetos 
de oraciones significativas, y no obstante, una vez que estas oraciones se analizan con toda precisión resulta que dejan de tener tales sujetos. ${ }^{8}$

Desde su primera redacción la teoría de las descripciones se presenta vinculada a las nociones de existencia y no existencia. Es característico y digno de nota que estas nociones se hallen desde un principio asociadas a la distinción que establece Russell entre "conocimiento directo" del objeto (acquaintance) y conocimiento "acerca del" objeto al través de sus propiedades (knowledge about). La distinción corresponde a la que se presenta entre la función epistemológica del "nombre propio" y la que es propia de las "descripciones". Trataremos a continuación sucesivamente del nombre propio y de la descripción en el sentido de la primera de las tesis antes enunciadas y en cuanto el asunto nos permita hacer patente la noción justa de existencia. El curso de la exposición deberá conducirnos a la segunda de las tesis.

En el desarrollo de la primera de las tesis señaladas se hará notar cómo el concepto de existencia es un concepto lingüístico que no puede aplicarse al nivel del nombre propio lógico, y se presentará en su lugar la interpretación de las descripciones definidas como símbolos incompletos, pues, como lo hace ver Simpson, la idea de que las descripciones no significan nada independientemente se apoya en el supuesto epistemológico de que "si una expresión es significativa, entonces su significado es la denotación".

Russell define el nombre propio algunas veces desde el punto de vista exclusivamente sintáctico. Otras veces lo define de acuerdo con la función epistemológica que desempe-

8 Cf. O. D. pp. 104-106, y M. PH. D., pp. 84-85. Estas dos tesis aparecen ya claramente destacadas por P. F. Strawson en su artículo "On Referring", reproducido en Essays in Conceptual Analysis, ed. A. Flew, London, Macmillan, 1960, pp. 24-25. (On Referring se citará con las iniciales O. R.)

9 T. M. Simpson, Formas lógicas, Realidad y significado, ed. cit. p. 117. 
ña. El nombre propio se define sintácticamente como una palabra que solamente puede presentarse en una proposición como sujeto, o bien, como una palabra que puede presentarse en cualquier proposición que no contenga variables, pero que no denota ni predicado ni relación alguna. ${ }^{10}$ Para nuestro propósito esta definición podría parecer, a primera vista, menos importante que la definición epistemológica. Pero la definición sintáctica, si distinta, no necesariamente ha de pugnar con su definición epistemológica. En efecto, la definición epistemológica del nombre propio considera la palabra que funciona como nombre en su referencia directa a un individuo, en cuya denotación inmediata descansa su significación y sólo en eso, es decir, independientemente de lo que puedan significar otras palabras con que eventualmente aparezca enlazado. El nombre propio, dice Russell, "es un símbolo simple que designa directamente a un individuo el cual es su significación, y que tiene este significado propio independientemente del significado de las otras palabras". ${ }^{11}$ En la medida, pues, en que el nombre propio reduce su significación a esta referencia inmediata al individuo del cual es el nombre, su definición sólo puede ser una definición ostensiva. Por el otro lado, en la medida en que su significación es independiente de las demás palabras con que pueda enlazarse, no es posible hacerla manifiesta por medio de una definición verbal. Po. demos decir, entonces, que los nombres propios tienen una manera autárquica de significar, que significan sólo porque hacen presente al individuo. Pero esta definición epistemológica, decíamos, no pugna, sino que puede concertarse simultáneamente con el papel que le asigna su definición sintáctica, pues el nombre propio al mismo tiempo que sólo

10 B. Russell, Human Knowledge, its scope and limits, New York, Simon \& Schuster, 1948, p. 73: el nombre propio es "una palabra que nunca puede presentarse en una proposición sino como un sujeto o término, o bien: un nombre propio es una palabra que puede presentarse en cualquier forma de proposición que no contenga variables". Una definición semejante se puede ver en M. PH. D., p. 167.

11 B. Russell, Introduction to Mathematical Philosophy, London, Allen \& Unwin, 1960, p. 174. (Se citará con las iniciales I. M. PH.). 
puede ordenarse como sujeto de una proposición sólo puede significar a un individuo que nombra directamente. En otras palabras, si el nombre propio interviene como sujeto de una proposición que no contiene variables es porque denota algo que conocemos de un modo inmediato, y viceversa.

Partiendo de lo que se acaba de indicar es posible efectuar un primer acercamiento al sentido que tiene para $\mathrm{Ru}$ ssell el término "existencia", si bien este primer encuentro se verificaría exclusivamente por via negationis, es decir, conduciría al punto en que no es posible hablar de la existencia. Es evidente que si un nombre propio se presenta ya aisladamente o ya en una proposición que no contiene variables, siempre es el símbolo de un existente, de un individuo existente, pues lo característico del nombre propio está en que su significación se agota en la referencia directa e inmediata al individuo que nombra, de suerte que, a menos que exista el individuo que denota, el nombre no puede tener significación. ${ }^{12}$ Es preciso advertir, sin embargo, por qué aun cuando el nombre propio denota un objeto existente no logra ser la vía adecuada para ofrecernos el verdadero o legítimo concepto de existencia. Cierto que el nombre propio designa por sí mismo un objeto existente, pero, según Russell, nunca el nombre propio dice que existe el objeto nombrado; se limita a designar el objeto inmediatamente presente sin implicar nada más acerca de él, sin implicar respecto del objeto que nombra ninguna otra propiedad, así sea la de que existe o que no existe. La razón por la cual no podemos decir de un objeto nombrado que existe o que no existe es que, si expresamos " $a$ existe" o " $a$ no existe", donde $a$ es el símbolo de un nombre propio, estas expresiones equivalen a decir que la propiedad de ser existente se aplica o se predica acerca de un individuo que ya está ahi antes de toda predicación puesto que he comenzado por

12 I. M. PH., p. 178-179: 'La proposición 'el tal y cual existe' tiene sentido sea verdadera o falsa; pero si $a$ es el tal y cual (donde $a$ es un nombre), la expresión ' $a$ existe' no tiene sentido... porque, si $a$ es un nombre debe designar algo: lo que no designa alguna cosa no es un nombre y, por lo tanto, si intentó ser un nombre. es un símbolo carente de significado". 
nombarlo demostrativamente, o que la propiedad de no existir se predica de un individuo que por su parte existe puesto que lo he nombrado. En el primer caso el predicado de existencia no dice nada, es supérfluo; en el segundo caso tampoco, es contradictorio. El concepto de existencia, según esto, sólo puede alcanzarse ahí donde sea posible hablar de existencia, y por lo pronto es imposible hablar de existencia al nivel del nombre propio y lo nombrado. "'Existencia' y 'ser', si han de significar algo, tienen que ser conceptos lingüísticos que no pueden aplicarse directamente a los objetos". ${ }^{13}$

Tenemos entonces el siguiente resultado: por nombre propio sólo debe entenderse la palabra sujeto de una proposición sin variables, que significa por el hecho de designar un individuo de un modo inmediato independientemente de lo que signifiquen otras palabras, esto es, por designar per se un objeto existe acerca del cual, sin embargo, nunca indica "que existe".

Ahora podemos decir que si el nombre propio sólo significa porque presenta inmediatamente un objeto individual, independientemente del contexto lingüístico en que pueda tener lugar, entonces no puedo analizarlo en otras palabras y sostener que se conserva el mismo significado del nombre en estos otros símbolos en que supuestamente lo habría analizado. El nombre propio no sólo es un símbolo simple en el obvio sentido de que las letras de que se compone ya no son símbolos, sino también en el sentido de que su significado no puede más analizarse en otros símbolos, por mucho que éstos parezcan significar lo mismo. Nos equivocaríamos totalmente, según Russell, si, queriendo analizar un nombre, pensaramos que "Scott", utilizado como nombre propio, significa lo mismo que "la persona llamada 'Scott", o "la persona que lleva el nombre 'Scott", , porque mientras que en el primer caso simplemente nombramos un

13 B. Russell, An Inquiry into Meaning and Truth, Penguin Brooks, 1962, pp. 61-62. Cf. Principia Mathematica, Cambridge University Press, 1960, pp. 174-175. (Principia se citará con las iniciales P. M.). 
individuo, en el segundo caso lo "describimos" como el individuo que lleva ese nombre. En el primero el nombre no forma parte del hecho afirmado, sino que es el símbolo para nombrar algo que es un hecho. En el segundo el nombre no sólo es parte de la afirmación, sino que también constituye el hecho que estamos afirmando. ${ }^{14}$ Así, no podemos desplazar por análisis un nombre propio, substituirlo por una descripción, y sostener que se conserva el mismo significado. Por tanto, tampoco se puede sostener que la proposición en cuyo sujeto se presenta un nombre propio siga siendo la misma proposición cuando en lugar de ese nombre establecemos una frase como "la persona (o el objeto) que se llama ...", o cualquier otra frase descriptiva, porque, si nombre y descripción no coinciden, su desplazamiento y substitución en el contexto de la proposición lleva consigo un desplazamiento y una substitución de una proposición por otra enteramente distinta. No podemos, pues, eliminar el nombre del contexto de una proposición y pensar que aún se trata de la misma proposición. Es en este sentido que Russell interpreta el nombre propio como un genuino constituyente de la proposición en que se presenta; lo que se puede expresar del modo siguiente: es imposible que por análisis de una proposición hagamos desaparecer el símbolo que designa directamente a un individuo existente sin que la misma significación proposicional desaparezca también. $\mathrm{O}$ el objeto nombrado existe, esto es, o el nombre es nombre propio, y entonces es un constituyente lógico de la proposición en que aparece como sujeto, o no es éste el caso, y entonces no es un constituyente auténtico de la proposición. ${ }^{15}$ Sólo el nombre propio puede tener significación con

14 I. M. PH., p. 175: "Cuando a un nombre se lo emplea directamente para indicar simplemente aquello de que hablamos, él no forma parte del hecho afirmado, o de la falsedad, si acontece que nuestra afirmación resulta ser falsa: sólo forma parte del simbolismo por medio del cual expresamos nuestro pensamiento".

15 En O. D., p. 114, podemos leer: "en toda proposición que podemos comprender (es decir, no sólo en aquellas de cuya verdad o falsedad podemos juzgar, sino en todas aquellas acerca de las cuales podemos pensar), 
independencia de cualquier contexto proposicional, pero si se integra en un contexto semejante sólo el nombre propio es un constituyente lógico de la proposición, un sujeto lógico y no sólo un sujeto gramatical.

Ahora bien, según Russell, hay una brusca distinción entre el nombre propio y las frases descriptivas que se utilizan para "caracterizar" algo (en este caso, naturalmente, cuando y si algo caracterizan, pues las frases en cuestión pueden ser vacías también); por ejemplo, "el autor de Waverley", y en general todas aquellas frases que se inician con el artículo determinado singular "el" o "la". El sentido recto de estas frases, a diferencia del nombre propio, está en que cuando y si expresan ciertas propiedades acerca de algo sólo pueden establecer esta referencia en virtud de su forma, es decir, por el significado que tienen estas frases en virtud de la definición contextual que se da de los artículos determinados "el", "la", pero no en virtud de una presentación inmediata, directa, del objeto. Es claro que podemos conocer acerca de un término descrito, "el tal", cuántas puedan ser las proposiciones que utilicen como sujeto una frase descriptiva, sin que por eso conozcamos quién o cuál es real y efectivamente "el tal", esto es, sin que conozcamos una proposición de la forma " $x$ es el tal", donde " $x$ " sea un nombre propio ("el hombre que consumó el crimen es alto" puede ser un ejemplo)..$^{16}$ La distinción que se da entre nombre propio y descripción corresponde, como se anticipó, a la distinción entre "conocimiento directo" y "conocimiento acerca de". Partiendo de esta drástica distinción es evidente que nada de lo que se ha declarado sobre el nombre propio puede ser aplicado a las descripciones. De las frases descriptivas no podrá sostenerse que

todos los constituyentes son realmente entidades de las que tenemos un conocimiento inmediato". Cf. P. M. pp. 51 y 56.

16 O. D., p. 103. Y en I. M. PH., p. 278: "es posible tener un amplio conocimiento acerca de un término descrito, por ejemplo, conocer muchas proposiciones acerca de 'el tal y cual', sin conocer realmente lo que el tal $y$ cual es, verbigracia, sin conocer ninguna proposición de la forma ' $x$ es el tal y cual', donde ' $x$ ' es un nombre'. 
impliquen referencia inmediata a un individuo; por lo mismo, no suponen denotación alguna de un existente en el sentido en que la existencia se halla esencialmente vinculada al nombre propio. Además, ni es posible que signifiquen algo independientemente del contexto proposicional en que puedan ocurrir, ni representan un constituyente lógico de las proposiciones cuando llegan a formar una de sus partes.

La razón de mayor peso que Russell aduce para probar que una frase descriptiva es siempre incomparable con un nombre propio, que no significa cosa alguna en sí y por sí misma, ni puede ser un constituyente cabal de las propo. siciones, puede presentarse de un modo conciso como sigue: si la frase descriptiva "el autor de Waverley" significara cualquier otra cosa, menos "Scott", la proposición "Scott es el autor de Waverley" tendria que ser necesariamente falsa, pero esto sería a su vez falso. Por otra parte, si la frase "el autor de Waverley" sólo significara "Scott", tendría que admitirse entonces que la proposición "Scott es el autor de Waverley" significa lo mismo que "Scott es Scott", lo cual tampoco es exacto, porque "Scott es el autor de Waverley" no expresa una tautología. El resultado es que, si la frase "el autor de Waverley" no significa "Scott" ni otra cosa cualquiera, en modo alguno puede decirse que comporte alguna significación, es decir, la frase "el autor de Waverley" no significa, por sí misma, nada. ${ }^{17}$

Este resultado trae consigo toda la doctrina del significado que sustenta Russell, la de los nombres propios y las descripciones. Pero si el nombre propio no agotara su significación en denotar directamente un individuo, sino que implicitamente o en forma no analizada tuviera el mismo sentido que comporta explícitamente o en forma analizada la frase descriptiva, y si, además, la frase descriptiva significara o denotara de un modo indirecto (al través de propiedades) el mismo individuo que el nombre propio denota directamente, en este caso nombre propio y descripción de-

17 Cf. P.M., Vol. I, p. 67. I.M.PH., p. 174. 
finida significarían o denotarían el mismo individuo, pero de un modo distinto, aquél de un modo directo y ésta de un modo indirecto, y tendrían el mismo sentido, pero de un modo distinto, aquél de un modo implícito, no analizado, y ésta de un modo explícito o analizado. Esto supondría que "el autor de Waverley" y "Scott" significaran o denotaran lo mismo, pero de un modo distinto, y que tuvieran el mismo sentido, aunque expresado de un modo distinto, que entre ambas expresiones no hubiera lugar para una tautología y que quedara suprimida la exclusión entre nombre propio y descripción definida quedando también abierta la posibilidad de que los nombres propios puedan tratarse como descripciones, como lo ha hecho Quine, y de que las descripciones definidas puedan tratarse como nombres propios, si se dispone de las constantes predicativas pertinentes, como lo ha hecho Richard M. Martin, por ejemplo. ${ }^{18}$ En todo caso la conclusión que saca Russell implica, en primer lugar, que cualquiera que sea la proposición que contenga una des. cripción nunca puede ser equivalente a la proposición que resulta cuando aquella descripción se substituye por un nom. bre, aun cuando este nombre designe lo mismo que la descripción describe, porque la proposición resultante o es necesariamente falsa o es una tautología, mientras que la proposición original sólo puede ser verdadera o falsa. Y en segundo, que esa situación es justo la prueba de que la frase descriptiva no se refiere, aisladamente, a ningún objeto.

Anticipemos que la frase descriptiva, en cuanto que no refiere ni presenta objeto alguno, no puede funcionar como sujeto lógico de una proposición ni ser un constituyente lógico de la misma en el sentido que se explicó, si bien puede suceder que aparezca en el lugar de la proposición que es propio del nombre. Cuando esto último ocurre las proposiciones pueden ser analizadas de tal suerte que, sin

18 Cf. W. O. Quine, Methods of Logic, London, Routledge \& Kegan Paul, 1966, pp. 215 ss. R. M. Martin, Truth and Denotation, London, Routledge \& Kegan Paul, 1958, Cap. II, F. pp. 54 ss. (Trad. esp. Madrid, Editorial Tecnos, S. A., 1962, pp. 65 ss.). 
que se pierda su significado, desaparezca la frase descriptiva como sujeto de la proposición, lo que mostrará que no es un sujeto lógico, sino un "sujeto gramatical" al que tampoco puede considerarse como un genuino constituyente de la proposición. Esto indica que, en contraste con el nombre propio, la frase descriptiva sólo puede significar y definirse dentro del contexto de la proposición en que se presenta, que no aisladamente. $\mathrm{Y}$ tal es lo que quiere decir Russell cuando declara que toda frase descriptiva es un símbolo incompleto, esto es, que las frases descriptivas únicamente pueden definirse en tanto que se las usa en una proposición. Naturalmente, si no son un constituyente lógico de las proposiciones queda por determinar qué es lo que significan cuando intervienen como un miembro de las proposiciones significativas.

Sea " $(7 \mathrm{x})(\Phi \mathrm{x})$ " el símbolo propio para expresar una descripción definida cualquiera- que puede leerse: "la $x$ que satisface $\Phi \mathrm{x}$ " o "el término $x$ que satisface $\Phi x$ ". Puesto que podemos conocer un número cualquiera, variable, de proposiciones relativas al objeto descrito sin que necesariamente conozcamos quién o cuál en particular es tal objeto, y puesto que la descripción definida describe siempre cierto objeto único y no más, toda descripción de esta clase reclama de una función proposicional " $\Phi x$ " y al mismo tiempo de la unicidad del valor que pueda satisfacer la variable " $\mathrm{x}$ ", es decir, del operador iota "( $7 \mathrm{x})$ ".

El análisis a que en seguida somete Russell las proposiciones en que se usa una descripción tenderá a mostrar lo que venimos anticipando, a saber, que toda proposición en cuya representación simbólica ocurre la expresión " $(7 \mathrm{x})$ $(\Phi \mathbf{X}) "$, puede expresarse en otra proposición en que este símbolo haya desaparecido sin que la significación se altere en lo más mínimo al pasar de una proposición a la otra. Sólo entonces puede revelarse qué es lo que significa la descripción en las proposiciones en que aparece, por qué la descripción no funciona como un constituyente lógico de la proposición, por qué es un símbolo incompleto, y cómo 
acaba por disiparse la confusión entre sujeto lógico y sujeto gramatical, en lo cual consistía la segunda de las tesis capitales de la teoría de las descripciones a que se aludió. "Siempre que se pueda suponer que el sujeto gramatical de una proposición no existe, sin que hagamos que la proposición pierda su significación, es claro que el sujeto gramatical no es un nombre propio, es decir, no es un nombre que representa directamente algún objeto. Así, pues, en todos esos casos la proposición debe ser susceptible de ser analizada de tal modo que lo que era el sujeto gramatical haya desaparecido". ${ }^{10}$ Para mayor claridad procederemos a presentar primero los resultados a que conduce el análisis de una proposición en que ocurre una frase descriptiva; posteriormente se observará, en términos de equivalencia, qué significa en definitiva esta proposición para que pueda expresarse en el modo en que lo hacen aquellos resultados, y por qué no puede expresarse más que como lo manifiesta dicho análisis. Estos pasos deben irnos dando simultáneamente el significado de "existencia" que propugna Russell.

\section{III}

A este propósito, se desarrollará en esta parte la segunda tesis enunciada, el análisis lógico del significado contextual de las descripciones definidas. Se puntualizará cómo el concepto legítimo de existencia, contra lo que sostiene Strawson, es parte del significado de las oraciones en que ocurre una descripción definida, y se fijará el sentido ontológico de existencia, vinculado al cuantificador existencial, como algo único que podría existir, puesto que la descripción se presenta en esas oraciones sólo como un argumento indeterminado de las funciones proposicionales.

Tomemos entonces un ejemplo de proposición en cuyo sujeto ocurre una descripción. Sea éste un ejemplo del propio Russell: "el autor de Waverley era poeta". En virtud

19 P. M., Vol. I, p. 66. 
de la descripción que ahí aparece, y de conformidad con lo que ya hemos convenido, esta proposición significa, en primer lugar, que "alguien" escribió $W$ averley. En efecto, esta proposición nunca podría ser considerada como verdadera si $W$ averley no hubiera sido escrito por lo menos por una persona; pero nada dice acerca de quién en particular sea tal persona. Es decir, la proposición implica una función proposicional como " $\Phi \mathrm{x}$ ", de la que es necesario afirmar que no siempre es falsa: por lo meuos en un caso es verdadera, y entonces implica $(\exists \mathrm{x}) . \Phi \mathrm{x}$, o sea, "hay al menos un objeto $x$, tal que $\Phi x "$.

Pero la proposición tiene referencia única, se refiere a una sola persona y no a más; entonces, cualquier otra persona ( sea, por ejemplo, $y$ ) que, además de $x$, hubiese escrito Waverley tendría que considerarse siempre como la misma persona $x$. Lo que la proposición significa, en segundo lugar, es que, dado que $x$ e $y$ hayan escrito $W$ averley, $x$ e $y$ serán siempre idénticos; y acerca de esto es preciso afirmar que siempre es verdadero, porque de lo contrario la proposición no podría tener referencia úlica. Así, lo segundo que la proposición significa es que $\Phi \mathbf{x}$. $\Phi y$. $\supset_{\mathrm{xy}} . \mathbf{x}=\mathrm{y}, \mathbf{o}$ sea, "si $\Phi \mathrm{x}$. $\Phi y$, entonces, para todos los valores de $x$ e $y, x$ es igual a $y$ ". Russell afirma, a propósito de esta última proposición, que si cualquier otro término tiene la misma propiedad $\Phi$ que tiene $x$ en la función proposicional " $\Phi \mathrm{x}$ ", y en tal caso resulta idéntico con este último, es porque uno y sólo uno, esto es, a lo sumo, un individuo satisface la función " $\Phi x$ ".

Ahora bien, es de este único término del que la proposición que se analiza afirma que "era poeta". Por consiguiente, lo que en tercer lugar significa la proposición es que, "si $x$ escribió Waverley, $x$ era poeta"; y no es menos necesario afirmar de ésto que siempre es verdadero, pues, como veremos, ya los dos momentos anteriores del análisis implican la existencia de un valor de $x$, y a menos que éste exista no será lícito atribuirle ninguna propiedad. Si, pues, siempre es verdadero que "si $x$ escribió $W$ averley, $x$ 
era poeta", también puede expresarse este significado diciendo "cualquiera que fuera quien escribió $W$ averley, era poeta". Pero entonces lo que implica el significado íntegro de la proposición es una doble función proposicional en la cual encontramos, en primer lugar, " $x$ escribió Waverley", y en segundo lugar, " $x$ era poeta". Si la propiedad que consiste en "escribir Waverley" y la que consiste en "ser poeta" no son más que dos casos que ilustran o determinan respectivamente las variables predicativas " $\Phi$ " y " $f$ ", la doble función proposicional que arroja el análisis de cualquier proposición del tipo que venimos exponiendo, debidamente enmarcada dentro del cuantificador existencial -la razón y el sentido del cuantificador se nos presentarán más adelante- será, en símbolos:

$$
(\exists \mathrm{c}): \Phi \mathbf{x} . \equiv \mathrm{x} . \mathbf{x}=\mathrm{c}: \mathrm{fc}
$$

Lo que se lee: "existe un objeto $c$ tal que ' $\Phi \mathrm{x}$ ' es verdadera cuando, y sólo cuando, $x$ es $c$, y 'fc' es verdadera", o bien. "existe un objeto $c$ tal que ' $\Phi x$ ' es siempre equivalente a ' $x$ es $c$ ', y $f c$ '. ${ }^{20}$

¿Qué relación guardan los resultados de este análisis con la idea de existencia? $O$ más precisamente, ¿qué idea de existencia se halla contenida en las proposiciones que acogen en su contexto una frase descriptiva y que el análisis haya puesto al descubierto? En el caso del nombre propio la existencia era un dato sobre el cual el nombre nada decía, acerca del cual nada podia afirmarse o negarse. Por otra parte, quedó establecido que la frase descriptiva, por sí misma, no hace referencia a ningún objeto, ni existente ni no-existente, que, considerada al margen de los contextos proposicionales, carecía de todo designatum susceptible de nombre. Pero se adelantó que, analizada en el contexto de una proposición, "algo" llegaría a significar. Entiéndase: "algo" llegaría a significar la proposición que la involucrara. Pues bien, lo que significan las proposiciones

20 Para los tres momentos del análisis que se acaban de exponer Cf. P. M., p. 68; y I. M. PH., pp. 177.178. 
que contienen en su contexto una descripción es algo que debe existir, para que puedan tener significado dichas proposiciones, pero cuya existencia es incomparable con aquella existencia que se asocia, más acá o más allá del lenguaje, a la significación del nombre propio. Así creemos entender a Russell, y en lo que sigue se trata de confirmarlo.

Resumamos lo que viene al caso. El primero de los dos momentos iniciales del análisis de la proposición reveló que por lo menos un individuo escribió Waverley, es decir, que la proposición "el autor de Waverley era poeta" será siempre falsa si jamás hubo al menos una persona que escribiera Waverley. El segundo momento del análisis mostró que, puesto que la descripción contextual es una descripción definida, de referencia única, era preciso que a lo sumo Waverley hubiera sido escrito por un individuo, y sólo por uno; de lo contrario, o la proposición es falsa o los "otros" que escribieron $W$ averley no son sino el mismo y único individuo, lo que confirmaría la unicidad de referencia y la verdad de la proposición.

Los dos pasos precedentes ya pueden colocarnos ante el tema y el concepto distintivo de existencia en Russell. En efecto, Russell declara que cuando se usan las descripciones en una proposición, el resultado a que conducen los dos primeros momentos de su análisis constituye ya un concepto, y a decir verdad el más consistente concepto, de existencia. Por lo tanto, que gracias a esos dos primeros pasos del análisis se puede ver con claridad que las proposiciones (I) " ' $x$ escribió Waverley' no es siempre falsa", y (II) 'si $x$ e $y$ han escrito Waverley, $x$ e $y$ son siempre idénticos", son equivalentes, juntas, a la proposición (III) "existe un término $c$ de tal manera que ' $x$ escribió $W$ averley' es siempre equivalente a ' $x$ es $c$ " "-donde 'c' es un valor o una constante que substituye a ' $x$ ' $y$ satisface a ' $\Phi x$ '. $O$ sea, que “ $(\exists x) .(\Phi x) " y$ “ $\Phi x . \Phi y . \supset$ xy. $x=y$ ”, son equivalentes, juntas, a "( $\mathrm{c}): \Phi \mathrm{x} . \equiv_{\mathrm{x}}, \mathrm{x}=\mathrm{c}$ ". ${ }^{21}$

21. Cf. Obras y páginas citarlas en la nota anterior. En la página 177 de 
Sin embargo, no acabaríamos por entrar en posesión de la idea de existencia que aquí aparece si no aclarásemos más las razones de fondo que tiene Russell para sustentarla. Queda entonces por explicar cuáles son en concreto estas razones.

Por los motivos que ya se señalaron una frase descriptiva podía expresarse mediante el símbolo $(\eta \mathrm{x})(\Phi)$. El examen de la proposición en que esta frase ocurre, o la definición de la proposición en que se usa el símbolo $\left(\eta_{x}\right)(\Phi x)$, nos condujo, en sus primeros dos momentos, a extraer el significado de que "al menos" y "a lo sumo" un individuo posee la propiedad de que habla la descripción, esto es, para volver a nuestro ejemplo, que "existe un término $c$ de tal manera que ' $x$ escribió $W$ averley' es siempre equivalente a ' $x$ es $c$ " ; expresándolo de nuevo en símbolos: ( c) : $\Phi \mathrm{x} . \mathrm{x}_{\mathrm{x}} \mathrm{x}=\mathrm{c}$. Pero la afirmación que se acaba de asentar no quiere decir otra cosa que lo que afirmamos cuando decimos: "el término que satisface la función ' $x$ escribió Waverley' existe". Expresemos esta última proposición, como hace Russell, mediante el símbolo “E! ( $\mathrm{\eta x})(\Phi \mathrm{x})$ ”. Lo que expresa este símbolo, podemos concluir, se encuentra ya incluido en toda proposición en que ocurre una frase descriptiva. Mas esto es justo lo que ha quedado definido como que "existe un término $c$ de tal manera que ' $x$ escribió $W$ averley' es siempre equivalente a ' $x$ es $c$ ',". De acuerdo con esta equivalencia se puede ver entonces que el resultado a que conducen los primeros dos momentos del análisis de las proposiciones que hablan acerca de una descripción no es otro que el siguiente:

Definición (A) $\mathrm{E} !(\eta \mathrm{x})(\Phi \mathrm{x}) .=:(\exists \mathrm{c}): \Phi \mathrm{x} . \equiv_{\mathrm{x}} \mathrm{x}=\mathrm{c}$

Ahora bien, por lo que se refiere al tercero de los momentos del análisis de la proposición, a saber, "el término

la I. M. PH. se dice: las proposiciones primera y segunda son equivalentes, juntas, a: "existe un término $c$ tal que ' $x$ escribió $W$ averley" es verdadera cuando $x$ es $c$, y falsa cuando $x$ no es $c$ ". En otras palabras, "existe un término $c$ tal que ' $x$ escribió $W$ averley" es siemore equivalente a ' $x$ es $c$ '". 
que satisface la función ' $x$ escribió Waverley', satisface la función ' $x$ era poeta'", lo cual se definió como "existe un objeto $c$ tal que ' $\Phi \mathrm{x}$ ' es siempre equivalente a ' $x$ es $c$ ', y $f c ":(\exists \mathrm{c}): \Phi \mathrm{x} . \equiv_{\mathrm{x}} \mathrm{x}=\mathrm{c}: \mathrm{fc}$, puesto que en toda proposición en que ocurre una descripción ya se halla contenida la afirmación de existencia que expresa el símbolo $E !(\eta \mathbf{x})(\Phi x)$, la siguiente expresión, al mismo tiempo que implica la equivalencia de la definición (A), asume en términos también de equivalencia el significado íntegro de la proposición:

Definición (B) : $\mathrm{f}[(7 \mathrm{x})(\Phi \mathrm{x})]=:(\exists \mathrm{c}): \Phi \mathrm{x} . \mathrm{x}_{\mathrm{x}} \mathrm{x}=\mathrm{c}: \mathrm{fc}$

Donde el miembro izquierdo de la equivalencia se lee "el término que satisface ' $\Phi \mathrm{x}$ ', satisface ' $\mathrm{f} x$ ' ", y el miembro de la derecha como ya se ha indicado. ${ }^{22}$

Se puede ver entonces, de acuerdo con los resultados obtenidos, que la afirmación de existencia es parte de lo que enuncian las proposiciones en que ocurre una frase descriptiva. En efecto, esto es lo que dejan establecido los dos primeros momentos del análisis, y como tal queda recogido en la definición (A) y asumido en la defición (B). Estos resultados, como es bien conocido, han sido objetados después por Strawson. Según Strawson, decir "el actual Rey de Francia es sabio" no es aseverar que existe una persona. y sólo una, que tiene la propiedad de ser Rey de Francia y la propiedad de ser sabio, porque esto sería cierto sólo si se hiciera uso de esa oración para referirse en circunstancias apropiadas a una persona particular. La oración misma como tal no enuncia o afirma que existe la persona. Si esa oración está usada en algún contexto particular, en una circunstancia espacio-temporal bien determinada, constituye una aserción existencial; en caso contrario no afirma, sino que se limita a presuponer, que existe la persona en cuestión. Así, cuando una oración como "el actual Rey de Fran.

22 Para las equivalencias de las definiciones (A) y (B) Cf. P. M., Vol. I, pp. 30, 67-68 y 173-174. En la página 68 expresa Russell en relación con lo que venimos diciendo " $E$ ! $(\neg x)(\Phi x)$ ' debe ser parte de lo que se afirma por cualquier proposición que verse cerca de $(7 x)$ ( $\Phi x)$ ". 
cia es sabio" se la usa para referirse a una persona, esta oración no constituye una aserción a menos que exista la persona a que directamente se refiere, porque cuando ésta no existe no puede decirse que se haya hecho uso de la oración. Lo que, como se recordará, es llevar la problemática de la existencia y el nombre propio russelliano a la esfera de las aserciones ¿cómo podemos afirmar que existe el individuo a que se refiere una oración en el uso cuando el uso mismo de la oración establece existencia? Pero por el momento sólo nos interesa la crítica de Strawson.

Como el análisis a que somete Russell aquellas oraciones no toma en cuenta el uso circunstancial de las mismas, piensa Strawson, tampoco es posible que ponga de manifiesto afirmación alguna de existencia como parte de las oraciones que analiza, por más que en ellas esté presupuesto que, por ejemplo, existe el Rey de Francia, o sea, que si "alguien pronuncia ahora la oración, estaría haciendo una aserción verdadera sólo si de hecho existiera actualmente un Rey de Francia, y sólo uno, y si éste fuera sabio". ${ }^{23}$ En consecuencia, si se considera que una de las funciones del artículo determinado "el" es la de ser señal de que se hace una referencia única, una oración como "el actual Rey de Francia es sabio" sólo indica, pero no asevera, que existe este individuo particular. 'Por lo tanto, usar la palabra 'el' de este modo es implicar [imply, pero en el sentido de entrañar, presuponer] ...que las condiciones existenciales descritas por Russell se cumplen. Pero usar 'el' de este modo no es aseverar que se cumplen esas condiciones". ${ }^{24}$

Pero ya quedó establecido que las frases descriptivas (y las oraciones en que se presentan) expresan ciertas propiedades acerca de algo -así, con toda indeterminación-, y que tienen esta referencia sólo en virtud de su forma, esto es, en virtud de la definición contextual que da Russell de los artículos "el", "la", pero no en virtud de una presen-

23 O. R., p. 33.

24 O. R., p. 36. 
tación inmediata, directa, del objeto. Entonces, la existencia que se manifiesta como parte de lo que afirman las oraciones que analiza Russell sigue siendo "algo" único indeterminado como resultado de una definición $\mathrm{y}$, por lo tanto, una construcción de un concepto semánticamente puro que hace abstracción, por las razones ya expuestas en la parte II, del uso circunstancial del lenguaje. "Mi teoría de las descripciones, advirtió Russell, jamás fue proyectada como un análisis del estado de ánimo de los que pronuncian frases que contienen descripciones" ${ }^{25} \mathrm{El}$ concepto de existencia que, según Russell, es parte de lo que afirman las oraciones en que figuran las frases descriptivas es un concepto estrictamente semántico. Pero el concepto de existencia por el que aboga Strawson es un concepto pragmático, puesto que se aplica solamente a los designata del lenguaje-objeto, del lenguaje que requiere, para decirlo con palabras de Strawson, "el tiempo, el lugar, la situación, la identidad del que habla, el asunto que constituye el foco inmediato del interés, y las historias personales del que habla y de aquellos a que se dirige". ${ }^{26}$ Strawson es consciente de la diferencia que medía entre un concepto de existencia y otro. Pero esto no parece impedirle incurrir en una subrogación, es más, en una confusión de conceptos, cuando niega que al realizar un análisis lógico de las oraciones en cuestión Russell haya podido poner de manifiesto como parte de las mismas una afirmación de existencia en el sentido pragmático que él propugna, pues esto supone, equivocadamente, que tal era lo que se pretendía - Russell, llegó a decir Strawson, se esfuerza "desesperadamente", como Leibniz, por llevar la lógica formal, las definiciones que prescinden de los requerimientos contextuales, a un campo totalmente diferente, al campo de las "afirmaciones de hecho". ${ }^{27}$

Pero del mismo modo que Strawson pudo decir, en ese equivocado supuesto, que era un absurdo sostener que tales

25 M. PH. D., p. 243.

26 O. R., p. 42.

$\because$ O. R., p. 43. 
oraciones constituyeran aseveraciones de existencia en sentido pragmático, se puede decir que sería también un absurdo negar que esas oraciones constituyan aseveraciones de existencia cuando se está considerando "existencia" en el marco de una definición lógica, lo que, sin duda, no hizo Strawson. Pero la crítica -de Strawson a Russell habría sido concluyente si hubiera llegado a establecer que se puede negar esto último en el caso del análisis.que efectúa Russell de estas oraciones sin sucumbir al absurdo, porque entonces habría probado que el análisis lógico de Russell era incorrecto.

En todo caso, la parte decisiva del análisis de la proposición en cuestión que termina en las definiciones (A) y (B) consiste en ser la prueba de que si el símbolo de la descripción " $(7 \mathbf{x})(\Phi \mathbf{x})$ " aparece como sujeto de una proposición, siempre es posible presentar esa proposición en forma tal que dicho símbolo haya desaparecido conservándose no obstante el mismo significado en una doble función proposicional ligada al cuantificador existencial. Esto es posible, digámoslo una vez más, en virtud de que la descripción definida que ocurre en la proposición no es un constituyente lógico de la misma. Otra forma de afirmar lo mismo, y en definitiva esta sería la razón de por qué se presenta en el definiens de las definciones (A) y (B) el símbolo del cuantificador existencial $\mathrm{J}$, consiste en decir que el símbolo de la descripción no puede ser tratado nunca como un valor de la función en que se presenta, de lo contrario, como señala Russell, funciones que son siempre verdaderas deberíamos esperar que fueran también siempre verdaderas respecto de una descripción. Pero no es el caso. Así, por ejemplo, siempre será verdadero que $(\mathrm{x}) . \mathrm{x}=\mathrm{x}$, donde $x$ puede ser substituida por un valor determinado o por un nombre propio; pero esa función no será siempre verdadera si $x$ se substituye por una descripción, digamos, por "el autor de Waverley", porque para que la función resulte verdadera en este segundo caso es necesario, además, 
que el autor de Waverley exista. Tampoco puede ser siempre verdadero que:

$$
(x) . f x \cdot \supset . f(\eta x)(\Phi x)
$$

porque si bien la función $(\mathrm{x})$. $\mathrm{fx}$ es siempre verdadera, puede resultar falsa respecto de la descripción, es decir, puede ocurrir que la descripción no describa nada, o lo que es lo mismo, que $(\neg x)(\Phi x)$ no exista. El condicional sólo pude ser válido cuando el término que satisface la función ' $\Phi \mathrm{x}$ ' existe, esto es, cuando $\mathrm{E} !(\eta \mathrm{x})(\Phi \mathbf{x}) .^{28}$

$¿ Q u e ́$ quiere decir, según esto, "el término que satisface la función ' $\Phi x$ ' existe"? Por lo que acaba de indicarse "el término que satisface la función ' $\Phi x$ ' existe" sólo puede significar que el citado término posee la propiedad ' $\Phi$ ', pues en el caso contrario la descripción no describe nada y el término que debería satisfacer la función ' $\Phi x^{\prime}$ no existe. Russell llega así a equiparar la existencia de un término descrito con la posesión de ciertas propiedades por parte de dicho término. El ejemplo es de Russell: puesto que el actual Rey de Francia no existe, no podemos afirmar que tenga la propiedad de ser calvo, o de no ser calvo. Cuando el término que satisface la función ' $\Phi x$ ' tiene alguna propiedad, cualquiera que esta sea, entonces existe. ${ }^{29}$

Pero debemos preguntar todavía ¿qué significa "existencia" cuando se trata de la existencia de un término en tanto que posee tal o cual propiedad? Esta cuestión se presenta ahora con toda la relevancia y pertinencia debidas. En efecto, admitamos que el término que satisface la función ' $\Phi \mathrm{x}$ ' tiene una propiedad $\Phi$. Ya no podemos afirmar que, puesto que dicho término existe si tiene tal propiedad, existe en el modo como existe el individuo designado por un nombre propio, porque el término de que aquí se trata no es el nominatum, sino el que posee aquella propiedad, el descriptum. Como un término descrito, según Russell, jamás implica el conocimiento acerca de qué o quién en particular posee

28 P. M., Vol. I p. 83. Cf. I.M.PH., pp. 175-176.

29 P. M., p. 174. 
la propiedad que hace al caso, mas, por otra parte, es necesario afirmar que dicho término existe, puesto que "al menos" y "a lo sumo" un individuo tiene esa propiedad, es menester concluir que el existente de que hablan las proposiciones en que se usa una descripción es un existente único que podría designarse directamente pero que realmente no está designado de esa manera. ${ }^{30}$ En consecuencia, esto significa que cuando decimos "el término que satisface la función ' $\Phi \mathrm{x}$ ' existe" - E! ( $\eta \mathrm{x})(\boldsymbol{\Phi x})$-, la descripción o su símbolo sólo puede funcionar como un argumento formal, indeterminado, de la función en que se presenta, ${ }^{31}$ para el cual podría haber un valor determinado que haga posible, o una proposición verdadera, en cuyo caso el valor del argumento es un existente, o una proposición falsa, y entonces tenemos el contrario. Es decir, la circunstancia de que la descripción o su símbolo sólo constituya un puro argumento indeterminado de una función conduce a interpretar "existencia" como aquello que, ontológicamente, podría acaecer en calidad de un valor único de dicho argumento. $\mathrm{Y}$ esto es justo lo que se significa con el símbolo " $\mathrm{E} !(\eta \mathrm{x})(\Phi \mathrm{x})$ ”. Esta era la noción de existencia de que se trataba cuando se dijo que en toda proposición en que ocurre una descripción ya se halla contenido $\mathrm{E} !(\eta \mathrm{x})(\Phi \mathrm{x})$ como parte de lo que afirma.

\section{IV}

Deslindado el concepto de existencia, en esta última parte se mostrará cómo es que, en rigor, sólo respecto de las descripciones puede afirmarse o negarse que algo existe; pero también se hará ver cómo no hay razones lógicas para sostener que las descripciones como "el cuadrado redondo" se refieren a objetos subsistentes.

30 La conclusión que aquí se obtiene desarrolla algunas opiniones ya vertidas sobre el tema por G. E. Moore. Véase de este autor: "Russell's 'Theory of Descriptions"', en Philosophical Pupers, London, 1959, pp. 187 y 193.

31 En efecto en P. M., p. 174, dice Russell: "Siempre que tenemos:

$\mathrm{E} !(2 \mathrm{x})(\Phi \mathrm{x}),(\mathrm{nx})(\Phi \mathrm{x})$ procede, formalmente, como un argumento ordinario para cualquier función en la que pueda ocurrir". El subrayado es mío. 
Partamos de las siguientes consideraciones. Si existencia significa aquello que podría acaecer como un valor determinado del argumento formal que constituye la descripción, entonces la frase descriptiva, como la proposición que la contiene, puede tener sentido aun cuando nada "real" se hallase referido por la descripción. Pero esto era lo que no ocurría con el nombre propio: aquí la referencia inmediata al objeto presente es la medida del significado. Hay, como podemos ver, un contraste tanto por lo que toca al sentido que puedan o no puedan tener nombre propio y descripción cuando se los refiere a la existencia, como por lo que toca a la existencia que estos símbolos implican cuando se los remite a la posibilidad de hablar o no hablar con sentido de ella. Porque mientras que no era posible hablar con sentido de que lo nombrado existe o no existe, a pesar de que si es nombrado existe, puede hablarse con sentido de que el descriptum de la correspondiente descripción existe o no existe, pues en este último caso queda abierta la posibilidad de que el valor que deba satisfacer el argumento formal de la descripción se haga presente o no, exista o no exista, quedando asi abierta la posibilidad de afirmarlo o negarlo como tal. Ante esta ambivalencia en la idea de existencia Russell habrá de pronunciarse por aquella que resulta de la posibilidad de hablar de existencia afirmándola o negándola, aun cuando esto suponga, teóricamente, el sacrificio de la existencia inmediata o directa. Por lo tanto, sólo de las descripciones puede ser, en sentido estricto, afirmada o negada la existencia. "Cuando en el lenguaje ordinario o en filosofía se dice que algo 'existe', siempre es algo descrito, es decir, no algo que se presenta inmediatamente, como una muestra al paladar, una mancha de color, sino algo como 'la materia', 'el espíritu', u 'Homero' (cuando se significa 'el autor de los poemas homéricos')".

Considérese el siguiente razonamiento: "Mi sensación presente existe; esto es mi sensación presente; por tanto, esto

32 P. M., p. 174. I. M. PH., pp. 178-179. 
existe". Russell sostiene que si bien las dos premisas de este razonamiento pueden ser verdaderas, la conclusión carece de sentido. ${ }^{33}$ En efecto, en una premisa describo " $\mathrm{mi}$ sensación" por una nota temporal y en cuanto afirmo que existe la afirmo como algo descrito; en la segunda premisa considero "esto" como lo que acabo de describir. Pero en la conclusión "esto" no está tomado como descripción, sino como símbolo designativo de un objeto inmediato, como nombre propio lógico, de suerte que no puede funcionar como un argumento indeterminado para una función proposicional respecto de la cual pueda presentarse la existencia como un caso. "Esto existe" es una adición ociosa.

Las razones que hemos expuesto explican por qué--cuando Russell define una proposición en que aparece como sujeto el símbolo de la descripición, cuando al definirla el símbolo de la descripción que se presenta en el definiendum desaparece en el definiens, siendo dicho símbolo no más que un argumento indeterminado cuyo valor está abierto a existir- el referido símbolo de la descripción, que se presenta como sujeto de la proposición, no es más que un sujeto gramatical que induce fácilmente a confundirlo con un sujeto lógico, es decir, con un símbolo designativo de un objeto, en el sentido fuerte del término, de tales o cuales propiedades.

Así, para volver a nuestro punto de partida, cuando decimos "el cuadrado redondo no existe" y expresamos en sím. bolos el sentido de esta oración del siguiente modo:

" $\sim E !\left(\eta_{X}\right)(\Phi x) "$, podríamos propender a pensar, como según Russell pensó equivocadamente Meinong, que "el cua. drado redondo" se refiere a un objeto, es decir, que constituye un sujeto lógico acerca del cual afirmamos luego que "no existe", cuando más bien deberíamos entender por esa oración que "no hay un valor que satisfaga con carácter de valor único la función ' $\Phi x$ ”'. Por lo tanto, si tomamos en cuenta que el símbolo de la descripción definida, y la noción de existen-

33 M. PH. D., p. 234. 
cia que con necesidad implica, quedó definido por ( $\mathrm{E}$ c): $\Phi \mathrm{x} . \equiv \mathrm{x} . \mathrm{x}=\mathrm{c}$, la oración "el cuadrado redondo no existe" es un caso de oración cuyo símbolo " $\sim \mathrm{E} !(\eta \mathrm{x})(\Phi \mathbf{x})$ " debería expresarse en un sentido más preciso de la forma siguiente:

$$
\sim\left[(E c): \Phi \mathbf{x} . \equiv x_{0} \mathbf{x}=\mathbf{c}\right]^{3+}
$$

El símbolo definido, en efecto, se presta a interpretarse equivocadamente como que "hay cierto objeto acerca del cual afirmamos que no hay tay objeto", mientras que el símbolo que lo define sólo expresa: "es falso que hay un objeto $c$ tal que $x$ tiene cierta propiedad cuando, y sólo cuando $x$ es igual a $c "$.

Los desarrollos precedentes habrán mostrado que cuando Russell define una proposición en cuyo contexto se da como sujeto una descripción, y cuando mediante su análisis pone de manifiesto que en ésta se halla implicado necesariamente el concepto de existencia, la existencia de que aquí se trata no es aquella que se vincula directamente al nombre propio y acerca de la cual no es posible afirmación alguna, sino la existencia que sólo puede afirmarse de las descripciones y que, cuando éstas se analizan, resulta ser un caso de función proposicional que es verdadera por lo menos $-\mathbf{y}$, cuando se refiere a una descripción definida, a lo sumo - para un valor de la variable. De aquí que el símbolo de la descripción desaparezca de la proposición que se define y que en su equivalente se presente la función respectiva precedida por el símbolo de operador existencial $E$, el cual no significa nunca un existente actual.

Por consiguiente, decir que algo existe equivale a decir, según Russell, que se da una función proposicional que es algunas veces verdadera, esto es, que existe por lo menos una proposición verdadera de la forma " $x$ es un tal" en la que $x$ es un nombre. ${ }^{35}$ La definición de existencia así expues-

34 P. M., p. 66.

$3 \tilde{5}$ I. M. PH., p. 172. 
ta se aplica exclusivamente a las descripciones indefinidas, donde existencia equivale a declarar que "dada una expresión $f x$ que contiene una variable $x$, y que se convierte en una proposición cuando se asigna un valor a la variable, ... la expresión $(\exists x)$. fx significa que hay al menos un valor de $x$ para el cual $f x$ es cierta". "Pero Russell advierte que esta definición de existencia también es válida respecto de las descripciones definidas con la condición de que el valor de la variable deba ser, en este caso, un valor único: la función aquí sólo puede ser verdadera para un valor de $x$ a lo sumo. ${ }^{37}$ La forma que aquí adopta el enunciado de existencia quedó ya expuesta con todas sus implicaciones, y se reitera ahora con toda sencillez: "hay un objeto (o un valor de) $c$ de tal manera que ' $\Phi x^{\prime}$ ' es siempre equivalente a ' $x$ es $c$ "'.

Insistamos, por último, en el aspecto, ciertamente paradójico, que se procuró destacar, a saber, que ni aplicada a las descripciones indefinidas ni a las descripciones definidas la noción de existencia alude, en cuanto tal, a un existente actual, sino a un objeto que podria existir. Para declararlo con Russell: 'cuando decimos 'hay' (uno o varios), no se sigue de la verdad de nuestra afirmación que lo que decimos que hay sea una parte del ajuar del mundo". ${ }^{38}$

36 M. PH. D., p. 234

37 I. M. PH., p. 172.

38 M. PH. D., p. 234. 
1. In the doctrine of Meinong it is established that phrases like "the round square" and "the mountain of gold", refer to objects which are not exactly existing objetcs. Although these objetcs do no exist, they are "something" and not simply a non-being. Such phrases do not only refer to nonexistents but allow the formulation of true propositions about these objetcs, as, for example, "the mountain of gold is golden and mountainous". If one cannot say that the objects to which these phrases refer exist, and if one can neither say that they simply reduce themselves to a nonbeing, then, supposing that they are something, the situation posed by Meinong's doctrine, is, apparently, contradictory; as Meinong himself came to express it: "there are objects for which it is valid that there are no such objetes".

The theory of Russell's descriptions is put forward as a reply to this type of paradoxes. In this principal objection, Russell shows that Meinong's doctrine goes against the principle of noncontradiction, since it maintains that there are objects which do not exist, which is like maintaining that the round square is round, and, also not round. But the reason that Meinong maintains that there are nonexistant objects lies in the distinction which he introduces between the subsistance of the object as such and the existence of the object as an actual object. Thus, when we say that there are objects (they subsist) in respect of which it is valid to say that there are no such objects (they do not exist), we perceive clearly that there is no such violation of the principle of non-contradiction.

Consequently, the problem presented to Russell by the doctrine of non-existent objects, within the theory of Meinong, is not so much logical as ontological, because the problem is rather that such objects can subsist and, subsisting, do not exist. Seen in this perspective, Russell would established the epistemological thesis that phrases such as "the mountain of gold", considered in isolation, have no meaning, refer to nothing. To believe the contrary, like Meinong, is to confuse phrases which can only have meaning in so far as they form a part of a proposition with symbols which signify Per se. Russell would also establish the logical thesis that those same phrases are apparent gramatical subjects, and not lo. gical subjects, correctly speaking. To maintain the opposite, as Meinong did, would be to confuse the logical function of the descriptive phrase with the logical function of the proper name. 
II. The epistemological definition of the proper name will be: it is the word that directly denotes an individual in whose denotation lies its meaning; its significance is independent of the word with which it eventually appears bound up. But Russell also gives a syntactic definition of the proper name. In this other definition the proper name is the word which can only figure as subjects in the proposition; that is, the word which may be presented in any proposition that does not contain variables. In fact, while the proper name can only be placed as the subject of the proposition it can only have meaning in so far as it directly denotes an indivi. dual, and vice versa.

From this, one makes the first approach toward the meaning of "existence" in Russell, although only by a via negationis; it is not possible to speak of existence at the level of the proper name. As a symbol whose meaning does not extend beyond the immediate denotation of an individual, the proper name is always the symbol of an existent. Therefore, to say " $a$ exists" or " $a$ does not exist", where " $a$ " is the symbol of a proper name, is equivalent to saying nothing, because " $a$ exists" expresses in reality "the existent exists", which is superfluous, whilst " $a$ does not exist" expresses the same as "The existent does not exist", which is contradictory.

Thus, "existence" is a concept that cannot be applied to the direct language in which the proper name opperates. This, the proper name, denotes an existent regarding which, however, it never indicates "that it exists".

In sharp contrast with the proper name, defined descriptions, such as "the man who committed the crime", on the one hand, have no direct reference to an individual, neither do they denote im. mediate existence in the sense of the proper name, nor, on the other hand, is it possible for them to signify in an independent man. ner. Firstly because, despite the fact that the described term is one and only one. we can know a variable number of propositions about a described term without knowing effectively who or what in particular that term is; and this is definitely what is expressed by the symbol of the defined description: " $(\eta x)(\Phi x)$ ". Secondly, because a phrase like "the author of Waverley" cannot signify the same as "Scott", since then "Scott is the author of Waverley", would be equivalent to the tantology of "Scott is Scott", which is false, nor can it signify something different from "Scott", since then "Scott is the author of Waverley" would have to be necessarily false. Therefore "the author of Waverley" does not signify either "Scott" or anything different from "Scott": that phrase for itself signifies nothing. The descriptive phrase itself is an incom. plete symbol, in the sense that, independently, it signifies nothing, 
and will only come to mean anything if it is used in a propositional context.

If the meaning of the proper name did not extend beyond direct. ly denoting an individual, as Russell maintains, but implicitly or in an un-analized form had the same sense which the descriptive phrase has explicitly or in analized form and if, moreover, the descriptive plırase denoted in an indirect way (by means of properties) the same individual that the proper name denotes directly, in this case the proper name and description would denote the same, but in a different way. In this case there would be no tautology between "Scott" and "the author of Waverley", and would leave open the possibility that the proper names could be treated as descriptions, and they as names.

The symbol of the defined description is introduced, and it is established how, by being the expression of an incomplete symbol, and by not being a logical constituent of the proposition, it can disappear when defining the proposition that it contains, showing that it is nothing more than a grammatical subject. This introduces the logical analysis of that proposition.

III. An example of a proposition in which a descriptive phrase figures as subject is "the author of Waverley was a poet".

(a) The first thing that the proposition signifies is that at least one person wrote Waverley, or that ' ' $x$ wrote Waverley' is not always false", in symbols: ( $E x), \Phi_{x}$.

(b) In accordance with the unique reference that by definition is ascribed to the definite article "the" with which the proposition opens, it refers at the most to a person. Therefore, what the proposition signifies secondly is that if $x$ and $y$ wrote Waverley, $x$ and $y$ are always identical, or that "if $\Phi x$. $\Phi y$, then $x=y$ ', is always true".

(c) It is from that single term that the proposition which one analizes affirms that "he was a poet". Thus, what the proposition thirdly signifies is that if $x$ wrote $W a v e r l e y, x$ was a poet. But then the integral meaning of the proposition is a double propositional function - in which we find " $x$ wrote Waverley" and " $x$ was a poet"- - duly placed within the existential quantifier, since the first two moments of the analysis reveal that there is at the least and at the most...

In symbols: $\left(\mathrm{E}_{\mathrm{c}}\right): \Phi_{\mathbf{x}} . \equiv_{\mathrm{x}} . \mathrm{x}=\mathrm{c}: \mathrm{fc}$

The important thing is that according to Russell, the proposition affirmed in (a) and that affirmed in (b) are equivalent together to the existential proposition "a term $c$ exists so that " $x$ wrote W'averley' is always equivalent to ' $x$ is $c$ ' -where $c$ is a value 
which substitutes $x$ and satisfies the function " $x$ wrote Waverley". But this existential proposition means no more than "the term that satisfies the function ' $x$ wrote Waverley' exists", which Russell expresses by the symbol $\mathrm{E} !\left(\eta_{\mathbf{x}}\right)\left(\Phi_{\mathbf{x}}\right)$. Thus, the affirmation of existence is now included in the whole proposition in which a descriptive phrase occurs; it is part of what these propositions affirm.

Strawson criticized these results by showing that to say "the pre. sent king of France is wise" is not to assert that such a person exists, but that in expressing that sentence one only presupposes that such a person exists. According to Strawson, the above sentence changes to an assertion of existence only if one uses it in clearly defined circumstances to refer directly or actually to that person. But in this case, how can we affirm that an individual exists to whom a sentence refers in the use, when the very use of the sentence already establishes its existence? Actually the concept proposed by Russell is a purely semantic concept, which disregards the circumstantial use of language, constructed from a definition of the definite article "the". The concept of existence which Stawson advocates is a pragmatic concept of existence from which it would be meaningless to judge the concept of existence proposed by Russell. Strawson's criticism of Russell's theory would have been conclusive if it had managed to establish its logical and semantic inconsistency.

Since, according to Russell, a describe term never implies knowledge of who or what in particular is the term which possesses the quality of which the description speaks, but, on the other hand, it is necessary to affirm that the said term exists, since, at the least, and at the most, an individual has that property, one has to conclude that the existent that the propositions mentioned in which a defined description is used, is a single existent which could be designated directly, but which in effect is not designated in this way. Thus, when we say "the term that fulfils the function ' $\Phi \mathbf{x}^{\prime}$ exists" $\mathrm{E} !(\eta \mathbf{x})\left(\Phi_{\mathbf{x}}\right)$ the description or its symbol can only function as an indeterminate formal argument of the function in which it is presented, for which there could or not be a determined value, i.e. an existent. Thus, "existence" is that which, ontologically, could occur as a single value of the said argument. It is of this notion of existence which one treats when one says that, in all propositions where a descriptions occurs, one will find $E !(\eta x)\left(\Phi_{x}\right)$ as a part of what is affirmed.

IV. If existence signifies what could occur as a value of the formal argument which constitutes the description, then the des- 
cription phrase or the proposition that contains it can have meaning even though nothing "real" is referred to by it. But this was not what happened with the proper name. Thus there is a contrast as much by what affects the meaning, which either can or cannot have a proper and description when they refer to existence which one atributes to the symbols, as with what affects the existence itself to which they are bound when they are open to the possibi. lity of speaking either with or without meaning. While it is not possible to speak with sense of whether the named exists or does not exist, one can speak with sense of whether the descriptum exists or does not exist, since in this last case there is the possibility that the value which should satisfy the formal argument of the description exists or does not exist, thus leaving the possibility of affirming or denying it. Therefore, it is only about descriptions, strictly speaking, that we can affirm or deny existence.

From this point of view "the round square does not exist" should not be interpreted as if "the round square" referred to an object about which we can then affirm that it does not exist, or as "there is a certain object (round square) about which we can affirm that there is no such object", but rather as "there is no single value which fulfills the function ' $\Phi x$ ' in the way of a single value".

Therefore, "existence" is equivalent to saying that given a function $f x$, which becomes a proposition when one assigns a value to the variable $x$, the expression $(\exists \mathrm{x})$. fx signifies that there is at least a value of $x$ for which $f x$ is true. This definition is valid for defined descriptions, but the value here is single.

What one has attempted to emphasize is that, paradoxical as it may be, the notion of existence applied to defined descriptions, does not allude as such to an actual existent, but to an object that could exist. 